\title{
Experimental realization of revival of silenced echo memory protocol in optical cavity
}

\author{
Mansur Minnegaliev*, Konstantin Gerasimov, Ravil Urmancheev, and Sergey Moiseev \\ Kazan Quantum Center, KNRTU-KAI, 420113, K. Marx Str. 10, Kazan, Russia
}

\begin{abstract}
We demonstrated a photon echo quantum memory for weak input optical pulses on the ROSE protocol in a $\mathrm{Tm}^{3+}: \mathrm{Y}_{3} \mathrm{Al}_{5} \mathrm{O}_{12}$ crystal placed in impedance-matched optical cavity. The quantum efficiency of $21 \%$ for a storage of time of $36 \mu$ s was achieved for single light pulses.
\end{abstract}

Active development of optical quantum technologies including optical quantum computing and long range quantum communications stimulates the creation of quantum memory (QM). The creation of highly-efficient QM will not only significantly expand the capabilities of these technologies, but also leads to fundamental impact on the creation of new directions in their development [1]. In last decade, there was proposed and experimentally realized a number of protocols of QM [2]. The schemes based on photon echo in solid state systems [3] demonstrated very promising results for achieving high quantum efficiencies and information capacity. Herein, the revival of silenced echo (ROSE) memory protocol seems especially attractive due to the possible use of natural inhomogeneously broadened line [4-6] that could significantly simplify practical implementation of QM. It was also recognized that the photon echo approach in free space schemes needs for a large optical density and requires backward spatial geometry for efficient quantum retrieval. However, these limitations can be eliminated by placing a QM cell in an optimal optical cavity $[7,8]$. Crystals doped by rare-earth ions are very attractive for this scheme due to its parameters of homogeneous and inhomogeneous broadening of the optical transitions [9]. By placing this crystal into impedance matched optical cavity we can increase the light-matter interaction up to complete absorption of input optical signals which leads to higher efficiencies $[7,8]$. In recent years there were several experimental demonstrations of photon echo QM in optical cavities $[10,11,12]$.

In this work for the first time we experimentally realized ROSE protocol in a $\mathrm{Tm}^{3+}: \mathrm{Y}_{3} \mathrm{Al}_{5} \mathrm{O}_{12}$ crystal placed in a concentric optical cavity. As in previous work [6] we used ${ }^{3} \mathrm{H}_{6}{ }^{3} \mathrm{H}_{4}$ optical transition $(\lambda=793 \mathrm{~nm})$ of thulium ions with concentration of $0.1 \%$ and orthogonal geometry for propagation of signal and control fields in the cavity. The concentric optical cavity was constructed from two spherical dielectric mirrors with reflection coefficients of $R_{1}=70 \%$ for the front (coupling) mirror and $R_{2} \approx 100 \%$ for back mirror. The overall cavity length was $\sim 90 \mathrm{~mm}$ which corresponded to $1.65 \mathrm{GHz}$ freespectral range. The impedance-matching condition for the ideal cavity with coupling mirror reflection $R_{1}$ and without any losses inside is achieved when weak total absorption $\alpha$ of the crystal with length $L$ satisfies $2 \alpha L=-\ln \left(R_{1}\right)$. In our case this condition is satisfied for $\alpha \mathrm{L} \approx 0.15$. So the experiment was performed on absorption line wing because $\alpha \mathrm{L}$ is close to

* Corresponding author: minnegaliev.mansur@yandex.ru 
1.4 at the line center. The crystal was placed in Montana Instr. closed-cycled cryostation and cooled down to the temperature $\mathrm{T}=4 \mathrm{~K}$. Typical behavior of the input light pulse and detected revived silenced echo is depicted in Fig. 1. One can see an absence of total impedance-matching condition because $7.5 \%$ percent of the input pulse was reflected. This could be caused by misalignment of the cavity. For no cavity case, the unabsorbed (reflected) signal could be $85 \%$ in this scheme. So the impedance-matched cavity provides 10 -fold enhancement of the signal absorption. Using this property, we obtained retrieval efficiency of $21 \%$ of input signal pulse for a storage time of $36 \mu$ s which is the best result for ROSE experiments and third in implementation of QM in optical cavity.

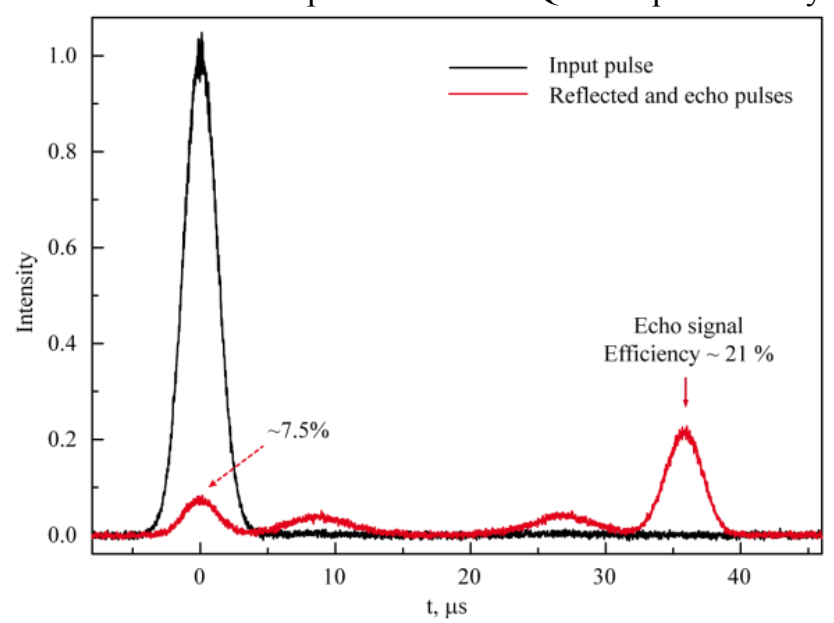

Fig 1. The signal of the revived silenced echo obtained by using $\mathrm{Tm}^{3+}: \mathrm{Y}_{3} \mathrm{Al}_{5} \mathrm{O}_{12}$ crystal in impedance matched optical cavity. The signals from scattered $\pi$-pulses (red curve) can be seen between the input pulse and echo signal. The echo signal efficiency was $21 \%$ with storage time $36 \mu$ s.

This work was supported by Russian Scientific Fund, Grant No. 14-12-01333 P.

\section{References}

1. F. Bussières, N. Sangouard, M. Afzelius, H. de Riedmatten, C. Simon, and W. Tittel, J. Mod. Opt., 60, 1519 (2013)

2. K. Heshami et al., J. Mod. Opt., 63, 2005 (2016)

3. W. Tittel et al., Las. Photon. Rev., 4, 244 (2009)

4. V. Damon, M. Bonarota, A. Louchet-Chauvet, T. Chanelière, and J.-L. Le Gouët, New J. Phys., 13, 93031 (2011)

5. S.A. Moiseev, Phys. Rev. A, 83, 012307 (2011)

6. K.I. Gerasimov, M.M. Minnegaliev, S.A. Moiseev, R.V. Urmancheev, T. Chanelière, and A. Louchet-Chauvet, Opt. Spectrosc., 123, 211 (2017)

7. S.A. Moiseev, S.N. Andrianov, and F.F. Gubaidullin, Phys. Rev. A, 82, 22311 (2010)

8. M. Afzelius and C. Simon, Phys. Rev. A, 82, 022310 (2010)

9. C.W. Thiel, T. Böttger, and R.L. Cone, J. Lumin., 131, 353 (2011)

10. M. Sabooni, Q. Li, S. Kröll, and L. Rippe, Phys. Rev. Lett., 110, 133604 (2013)

11. P. Jobez, I. Usmani, N. Timoney, C. Laplane, N. Gisin, M. Afzelius, New J. Phys. 16, 083005 (2014)

12. R.A. Akhmedzhanov, L.A. Gushchin, A.A. Kalachev, N.A. Nizov, V.A. Nizov, D.A. Sobgayda, and I.V. Zelensky, Las. Phys. Lett., 13, 115203 (2016) 